\title{
Prendre soin : contre qui ?
}

\section{Christiane Vollaire}

\section{(2) OpenEdition \\ Journals}

\section{Electronic version}

URL: http://journals.openedition.org/appareil/2346

DOI: 10.4000/appareil.2346

ISSN: 2101-0714

\section{Publisher}

MSH Paris Nord

\section{Electronic reference}

Christiane Vollaire, «Prendre soin : contre qui ? », Appareil [Online], Articles, Online since 23 September 2016, connection on 30 July 2020. URL : http://journals.openedition.org/appareil/2346 ; DOI : https:// doi.org/10.4000/appareil.2346

This text was automatically generated on 30 July 2020 .

\section{(c) (i) (9)}

Appareil est mis à disposition selon les termes de la Licence Creative Commons Attribution - Pas d'Utilisation Commerciale - Pas de Modification 4.0 International. 


\title{
Prendre soin : contre qui ?
}

\author{
Christiane Vollaire
}

\section{AUTHOR'S NOTE}

Pour les Hospices de Tonnerre

Conférence du samedi 10 septembre 2016

1 Ce qui caractérise la notion de care, dès que l'on tente de l'aborder, c'est son caractère parfaitement lisse et sans aspérité. Une sorte de gallet rond qui glisse entre les doigts et rebondit sur l'eau dans le parcours automatique d'un ricochet, ne donnant prise à peu près sur rien. Qui contestera en effet qu'il faille s'occuper d'autrui, prendre soin de soimême, rendre la société vivable, et toute cette enfilade de truismes par lesquels on définit la « bonne vie » à grandes lampées de moraline?

2 Et en quoi la réitération de ces discours plus moralisateurs que proprement moraux, dont la culture chrétienne est emplie à ras bord depuis ses origines, dont l'islam fait le terreau de l'obligation de charité qui est au centre de sa doctrine, dont la pensée humanitaire infuse de ses antécédents missionnaires $\mathrm{du} \mathrm{xvI}^{\mathrm{e}}$ siècle à ses débuts caritatifs de la fin du XIXe, et jusqu'aux campagnes publicitaires omniprésentes des ONG contemporaines, en quoi donc cette réitération lénifiante pourrait-elle constituer une pensée novatrice?

3 En rien, semble-t-il, du point de vue de son contenu : un concept aussi consensuel que celui de la sollicitude ne peut guère être interrogé, dans tout ce qui le caractérise comme absolument privé de cette rugosité qui conditionne l'énergie de la pensée. Il ne pourra donc être questionné qu'à partir de ce à quoi il s'affronte, ou de ce par quoi il est susceptible d'être instrumentalisé.

4 La question ne sera donc évidemment pas : faut-il être bienveillant, attentif, soucieux de l'autre? Ou de qui prendre soin et pourquoi? Car même les idéologies les plus violentes supposent cette forme de bienveillance. L'idéologie nazie pose bien une attention au bien-être de la race pure fragilisée par les menaces de décadence et de contamination; l'ultra-nationalisme serbe s'affirme bien comme un rempart 
protecteur, dans le souci d'une révérence émue aux traditions médiévales; et le pouvoir iranien actuel se manifeste dans le culte de ses martyrs de la guerre d'Irak et le soin accordé à leur mémoire, dans le temps même où il impose une omerta de l'ordre du négationnisme sur les massacres qu'il a commis dans ses prisons.

Le discours humanitaire lui-même, le plus unanimement médiatisé actuellement comme discours de l'attention portée à l'autre, a pris naissance, comme son histoire ne cesse de le montrer, dans le temps et par les acteurs de l'ultra-violence coloniale.

6 Et bien sûr les lieux de soin, depuis les origines, loin d'apparaître prioritairement comme des espaces de réalisation du care, se sont illustrés au cours de leur histoire comme les instruments de ce que Michel Foucault appellera, dans l'Histoire de la folie à l'âge classique publié en 1964 et réédité en 1972, «le grand renfermement » : un outil de surveillance et de contrôle de la France de Louis XIV au XVII siècle. Dans Les Corps vils, publié en 2008, le philosophe Grégoire Chamayou montre qu'ils étaient aussi des espaces de discrimination sociale en vue de la sélection des sujets d'expérimentation médicale. Et dans son rapport de 2015, la contrôleure des lieux de privation de liberté Adeline Hazan a clairement dénoncé un certain nombre d'hôpitaux psychiatriques comme étant des lieux de maltraitance caractérisée.

7 Ce sont donc plutôt deux questions qui nous occupent :

8 - Quelles forces faut-il combattre pour affirmer un « prendre soin»?

9 - Quelles violences la focalisation sur le « prendre soin » pourrait-elle viser à occulter?

\section{Un historique autour du genre}

10 Bien qu'elle trouve des sources incontestables dans les traditions religieuses et humanitaires que l'on a mentionnées, la notion de care a une histoire plus récente : elle n'apparaît de façon conceptualisée qu'au début des années quatre-vingt, avec le travail de la psychologue américaine Carol Gilligan. Celle-ci ne s'occupe ni du système hospitalier, ni de quoi que ce soit qui concerne le soin. Elle travaille bien plutôt sur les questions morales, comme élève d'un disciple de Piaget, Lawrence Kohlberg. Celui-ci a élaboré une théorie du développement moral, dégageant divers stades de l'acquisition des principes moraux, qui lui permet d'affirmer une discrimination de sexe dans la capacité d'acquérir ces principes : les filles atteindraient un stade moins élevé que celui des garçons.

11 C'est sur ce point que Gilligan s'oppose à lui : ce qu'il prend pour un niveau inférieur selon une appréciation quantitative serait bien plutôt une approche différente selon une distinction qualitative : une morale fondée davantage sur la nécessité empirique de la relation à l'autre. Une éthique de la discussion et de la négociation au cas par cas, plus affinée, plus soucieuse de l'intersubjectivité, caractériserait la tendance féminine, à l'encontre d'une éthique des principes et des valeurs, plus surplombante, qui caractériserait l'approche masculine. Il n'y aurait pas entre les deux une différence de degré, mais une différence de nature. L'ouvrage s'intitule In a Different Voice et sort en 1982. Il sera traduit quatre ans plus tard sous le titre Une voix différente. Il met en évidence le care comme cette exigence morale d'une voix différente, attentive au souci de l'autre, qui caractériserait le féminin.

12 C'est onze ans plus tard, en 1993, qu'une autre chercheure américaine, Joan Tronto, politologue, reprendra le terme dans une intention polémique à l'égard de Gilligan. Et 
la polémique portera précisément sur la question du féminin. Tronto fait valoir que le care ne saurait être considéré comme l'attribut originel d'un genre, mais au contraire comme une nécessité humaine, universellement présente en tous, que les traditions philosophiques majoritaires, celles du rationalisme triomphant, auraient occultée. Il faudrait donc rendre son espace d'expression à un concept qui d'une part relève d'un fondement anthropologique, et d'autre part a pleinement sa place dans l'espace public qu'il doit investir, après avoir été indûment dévalorisé comme assignable au seul espace privé. Non seulement le prendre soin n'est pas une tendance spécifiquement féminine, mais les activités qu'il génère doivent être pleinement reconnues et assumées dans une économie générale qui jusqu'ici n'a valorisé dans l'activité de travail que la part rigoureusement productive, au détriment de celle des services. En montrant que la prétention à attribuer le care au féminin (que ce soit dans la réalité du soin ou dans la théorie d'un mode de rapport au monde spécifiquement genré) est elle-même discriminante, Tronto fait basculer le care du domaine de la psychologie à celui de la sociopolitique. Le livre a été traduit en français en 2009, sous le titre Un monde vulnérable.

Le care apparait donc en France comme un concept des années deux mille, issu du monde protestant anglo-saxon de la fin du $\mathrm{xx}^{\mathrm{e}}$ siècle, de ses préoccupations sur l'empirisme et les valeurs morales, et de ses polémiques sur la question du genre. Le livre de la philosophe américaine Judith Butler, Trouble dans le genre, paru en 1990, interfère manifestement avec la critique élaborée par Tronto du livre de Gilligan, puisque ce dernier vise d'abord à dénaturaliser le care comme féminin pour dénoncer précisément le caractère genré des professions qui lui sont liées, du travail domestique du ménage au travail hospitalier des soins aux malades, en passant par la garde des enfants. Les chercheurs qui le convoquent aux EUA, au Canada ou au Royaume-Uni comme en France sont très majoritairement des femmes, ce qui donne à penser sur le caractère genré non seulement de ces pratiques, mais de la théorisation critique même de ces pratiques.

\section{Un rapport au politique indéterminé}

Ainsi, pour Tronto, dénoncer le machisme des traditions philosophiques qui ignorent le care reviendra à dénoncer une automutilation de la philosophie elle-même. C'est tout un pan de sa propre histoire que la philosophie méconnaît ainsi, et que le care peut lui permettre de seréapproprier. Le care trouve à ses yeux sa double filiation dans la pensée aristotélicienne, pensée de la réalité concrète du monde opposée au surplomb de l'idéalisme platonicien, et dans les courants empiristes de l'Europe des Lumières, sous l'égide des théoriciens écossais : Hutcheson, Hume et Adam Smith. Tous trois ont en commun de tenter de saisir un fondement anthropologique aux valeurs morales, ancrées dans les besoins humains fondamentaux, en leur refusant le statut métaphysique que les traditions idéalistes leur accordaient :

Avec quelques exceptions notables (David Hume, Adam Smith), la pensée politique occidentale a laissé de côté les questions ayant «traditionnellement informé la vie des femmes et des serviteurs / servantes, des esclaves et des travailleurs $»{ }^{1}$

Mais, paradoxalement, visant à intégrer le questionnement moral dans une problématique politique empiriquement fondée, Tronto fait preuve d'une étrange 
volonté d'aveuglement à l'égard des auteurs sur lesquels elle prétend se fonder. Ainsi écrit-elle à propos de la pensée aristotélicienne :

Dans sa conception du care, de la moralité concrète, de la nature réciproque de la citoyenneté et de la manière dont l'amitié met au cœur de notre vie l'attention à autrui, c'est bien un cadre théorique riche et gratifiant qu'Aristote offre à tous ceux qui ont un intérêt pour le care. ${ }^{2}$

Hommage à Aristote dont le " cadre théorique riche et gratifiant » ne porte que sur la dimension morale d'une œuvre qui est pourtant fondamentalement politique, alors même que c'est la dimension politique du care que Tronto prétend mettre en évidence.

Mais, surtout, alors qu'elle prétend valoriser chez Aristote la dimension empirique et quasi-pragmatique de son travail, et son rapport étroit au réel, cette phrase de préambule concernant son rapport à la réalité politique de son temps :

Ôtons les considérations périmées d'Aristote sur les esclaves, les travailleurs et les femmes. L'image qui en ressort est alors pleine de promesses. ${ }^{3}$

Autrement dit: ôtons d'Aristote tout ce qui concerne son rapport réel à la vie sociopolitique de son temps et aux rapports de pouvoir et de domination réels que sa pensée évacue ou justifie en tant que tels, pour examiner en quoi il pourrait être un précurseur de la pensée du care, et ce bien qu'il légitime pleinement la condition des esclaves, celle des travailleurs, celle des femmes... et, en bref, celle de tous les subalternes au sein de la cité. Une étrange méthode d'usage de la pensée d'Aristote à la fondation d'une politique du care.

Et pourtant, la question des subalternes, issue de la pensée post-coloniale, telle que la porte une auteure comme Gayatri Spivak, marque aussi la réflexion de Tronto. Théoricienne d'origine indienne enseignant aux États-Unis, Spivak a fait émerger le concept de "subalterne» comme vecteur de la pensée critique post-coloniale, en l'appliquant en particulier à la question du genre lorsqu'elle a publié en 1988 Can the Subaltern Speak?, qui sera traduit en français en 2006 sous le titre Les Subalternes peuventelles parler?

Une telle question croise la réflexion sur le care, et sur le travail silencieux, invisible ou dévalorisé dont il témoigne, mais aussi sur les processus d'exploitation auxquels il donne prise. Et Tronto montre que ces processus d'exploitation du travail du care sont au cœur d'une économie qui, pour être souterraine, n'en est pas moins réelle. Elle écrit ainsi :

Fisher et moi-même avons également décrit quatre phases du care [...] le fait de se soucier de quelqu'un ou quelque chose (caring about); de prendre soin de quelqu'un (caring for); de soigner quelqu'un (care giving); d'être l'objet du soin (care receiving).

[...] Cette définition regroupe plusieurs disciplines et perspectives que les chercheurs en sciences sociales ont tendance à distinguer le care : pour soi-même (le souci de soi) semble être une catégorie psychiatrique, le care pour les autres (le souci des autres) une catégorie sociologique et le care à l'égard du «monde» (le souci du monde) semble relever de catégories économiques et politiques.

[...] Ce qui m'intéresse toutefois ici, c'est de relever les différentes positions de pouvoir dans lesquelles se trouve celui qui offre les soins et celui qui les reçoit dans chaque situation. ${ }^{4}$

21 L'activité de prendre soin n'est ainsi pas seulement prise dans le sens moral de la sollicitude, mais dans celui d'une position sociale à l'égard de soi-même, à l'égard de l'environnement immédiat du milieu social et professionnel, et à l'égard de ce qu'Hannah Arendt appelait «le monde»: l'ensemble des relations politico- 
économiques que constituent actuellement les processus de globalisation. On le voit, la notion de care devient extrêmement inclusive : distordant chez Gilligan une notion issue $\mathrm{du}$ monde des affects et des émotions pour la faire entrer dans un questionnement moral de choix éthique et de comportement social, elle est encore réorientée chez Tronto pour intégrer une problématique liée généralement au monde du travail, et plus précisément aux modes d'exploitation dont il est le lieu.

Dans les deux cas, la notion consensuelle de care subit en sous-main une autre torsion : celle de la dimension polémique qui la sous-tend. Chez Gilligan, la polémique est axiologique : elle vise à revaloriser ce qu'elle estime être un caractère du féminin, à l'encontre du déni de valeur dont il fait l'objet dans la hiérarchisation morale élaborée par Kohlberg. L'émergence du concept de care affronte et combat une axiologie à la fois idéaliste et viriliste: un concept des valeurs morales fondé sur une métaphysique subreptice du devoir et de la "vertu», qui surplombe l'action, pour lui substituer une idéologie de la relation et une éthique de la discussion et de l'attention à la demande.

\section{La question des subalternes dans le monde post- colonial des migrations}

Chez Tronto, cette idéologie s'affronte à des formes de pouvoir politique : affirmer la valeur morale du care, c'est indissociablement revendiquer sa valeur économique. Prendre soin n'est pas seulement un désir psychologique ou une attitude éthique, c'est aussi un métier. Cela suppose d'une part que soit reconnue une configuration d'activités multiples dont une large part relève de l'espace privé. Espace de la nonreconnaissance publique, ouvert de ce fait même aux possibilités d'exploitation. Cet espace concerne depuis toujours, dans la plupart des sociétés, la catégorie des femmes auxquelles, comme le montrent en particulier les travaux de l'anthropologue Paola Tabet dans La Construction sociale de l'inégalité des sexes, paru en 1998, ne sont dévolus que les outils de l'activité subalterne et domestique. Le gynécée grec en est un exemple. Mais les activités domestiques, si elles concernent bien les femmes, concernent aussi celles qui ne font pas partie de la famille, mais de la domesticité, celle-ci s'établissant non seulement en termes de genre, mais aussi en termes d'appartenance "ethnique " et sociopolitique. Comme l'écrit la sociologue Pascale Molinier :

Ce sont ces activités de care « réservées » aux catégories subalternes qui focalisent l'attention, contribuant à marginaliser et à minorer l'importance du care. Il n'est donc pas contradictoire que de nombreuses femmes se reconnaissent psychologiquement dans l'éthique du care, et qu'il faille pourtant en affirmer politiquement la neutralité en termes de genre. [...] Le risque principal en l'occurrence est de rendre visibles les privilèges de certains. C'est pourquoi le care ne reste visible qu'en partie, une partie soigneusement circonscrite : aux femmes, aux pauvres, aux immigrés. ${ }^{5}$

La revendication des valeurs du care pourrait être en ce sens considérée comme un biais pour une double dénonciation : celle de la forclusion de l'espace privé, qui fait obstacle à un regard public sur les conditions de travail; et celle des circuits clandestins de l'exploitation du travail, des profits qu'ils permettent et des nouvelles formes d'esclavage qu'ils génèrent dans une économie globalisée. Les Philippines en fournissent un exemple récurrent. Tronto écrit :

Aux Philippines, une nation qui encourage l'émigration, huit milliards de dollars provenaient en 2004 de transferts de fonds depuis l'étranger. ${ }^{6}$ 

toujours accomplie en vue de prendre soin des autres? Marx avait déjà pointé chez Rousseau le caractère indéterminé, et par là même occultant, du concept de " contrat social », qui supposait une unanimité possible de la "volonté générale », c'est-à-dire des intérêts d'une société tout entière. Et il mettait en évidence le fait que tout concept unanimisant du corps social n'a pas d'effet performatif dans la réalité des sociétés, mais en a en revanche un très puissant pour euphémiser la violence réelle et en occulter la prégnance.

Et l'historienne américaine Evelyn Nakano-Glenn affirme :

Les Philippines, par exemple, fournissent aux Etats-Unis et à d'autres pays industrialisés l'un des plus gros contingents de travailleurs/ses du care souspayées. Bien que le nombre exact de ces travailleurs/ses soit inconnu, en raison de la grande proportion de migrants dont on perd la trace, hors des circuits officiels, on estime à près de dix millions le nombre de ces travailleurs/ses réparti-e-s dans cent vingt pays. ${ }^{7}$

La sociologue des migrations Bridget Anderson cite ainsi, dans un travail de terrain de 2000, une employée philippine à Athènes, montrant comment se traduit concrètement le travail du care dans les formes de l'esclavage moderne :

Tu travailles à la minute où tu ouvres les yeux jusqu'à la minute où tu fermes les yeux. Tu dois aller jusqu'au bout de tes forces pour finir ton travail... Et tu dois attendre que tes employeurs aillent se coucher parce que, même si tu as fini de travailler, par exemple tu as fini le repassage, couché les enfants ou les grandsparents, même si tu les couches à dix heures, il y a toujours d'autres membres de la famille debout. Alors, tu restes à te dire Oh si je vais dormir, ils risquent de m'appeler pour leur faire à manger ou leur donner un yaourt. Et même si tu dors, tu as quelquefois l'impression d'être toujours en service. ${ }^{8}$

\section{Un outil conceptuel faible}

Ainsi l'éthique du care, devenue politique du care, tente-t-elle de tenir la triple injonction d'une revendication féministe à l'égard du pouvoir masculin, d'une dénonciation post-coloniale à l'encontre des rapports de domination politique liés aux processus migratoires, et d'une revendication économique à l'encontre de la dévalorisation des professions de soin.

faibles, parce que sa position, quelque légitimes que soient ses revendications, engage toujours un rapport victimaire au monde, un appel au bon sens moral ou à ce que Sandra Laugier appelle « les politiques de l'ordinaire », dont l'imprécision et le manque d'ancrage précisément politique constituent le handicap principal. Qu'est-ce que l'« ordinaire » en tant que tel, pour des sujets dont le quotidien qui doit le constituer est sans commune mesure possible? Qu'est-ce que l'ordinaire d'une professeure de sociologie américaine en regard de celui de la semi-esclave philippine dont il transcrit la parole ? Et en quoi un terme aussi vague peut-il permettre d'entendre la violence des conflits et de pointer clairement les niveaux de responsabilité de cette violence?

Et s'il s'agit de "prendre soin ", comment ne pas voir que la violence faite aux uns sera Ainsi les politiques du care relèvent-elles aussi d'un double langage dans lequel le « nous » ne parvient pas à trouver son assise. Tronto écrit : 
Ce à quoi servent les immigrants et les personnes d'autres origines, c'est à prendre soin de "nous». [...] Les démocraties peuvent-elles survivre, si elles ne font de certains citoyens rien d'autre que des serviteurs ? ${ }^{9}$

Mais elle affirme dans le même temps :

Comme le care devient de plus en plus le travail de " populations multiculturelles ", il sera plus facile de les exclure de la citoyenneté. L'exclusion dérivant de la distinction entre le privé et le public qui a confiné le care en dehors de la vie publique pourrait désormais se retrouver sous la forme d'une exclusion fondée sur la distinction entre care et "activités de service». Le "care véritable " sera uniquement réalisé par les personnes « de souche », sans signe distinctif, alors que les «activités de service » seront marquées par des statuts raciaux, linguistiques, religieux et migratoires. ${ }^{10}$

Du care à l'« activité de service ", la distinction demeure, dans le texte, parfaitement indéterminée. Mais il est clair que le «nous » de l'auteure est déjà excluant, puisqu'il n'inclut ni l'un ni l'autre. Les serviteurs exclus du « nous » de la chercheure sont donc bel et bien essentialisés dans leur position domestique, dans le temps même où demeure une ambivalence sur leur statut de citoyenneté : affirmé dans la première citation, nié dans la seconde.

Mais en outre, cette indétermination de l'éthique du care porte aussi sur les confusions possibles entre l'attitude du care et les métiers du care. Par définition, les professions de santé font partie de ces derniers. Or ces dernières sont le lieu d'une différenciation majeure entre ceux qui s'occupent de traiter la pathologie et ceux qui s'occupent de soigner les patients. Et cette hiérarchie conduit précisément à faire des seconds les exécutants des premiers. Dans la formation des médecins, la question du soin, de l'attention aux patients, est, comme s'en plaignent les étudiants qui ont choisi cette profession dans une intention soignante, totalement secondarisée: les questions d'internat ne la mentionnent pas, et toute la sélection repose exclusivement sur un savoir mathématico-biologique qui évacue la relation au patient. Celle-ci, mise au rancart comme une perte de temps dans l'acquisition des savoirs qui permettent d'entrer en compétition, est de ce fait même dévaluée. Le sociologue Aaron Cicourel mentionnait déjà il y a vingt ans la manière dont la conduite des entretiens auxquels il avait assisté entre médecins et patients mettait en évidence une surdité de l'ensemble des médecins concernés non seulement à la demande des patients, mais de ce fait même aux informations que ceux-ci pouvaient leur fournir, et qui auraient pourtant été utiles dans l'élaboration du diagnostic et dans le choix du traitement.

Et dans le monde hospitalier contemporain, le primat de la décision administrative sur la décision médicale et thérapeutique, dans le management de la gestion des lits et du temps de travail des personnels conduit à un supplément de pression et de hiérarchisation qui, sous couleur d'une ambition d'efficacité économique, aboutit à une redoutable inefficacité thérapeutique, voire à une véritable contre-productivité. L'industrialisation du système hospitalier, l'émergence des grandes structures de soin, accroît encore considérablement cette contre-productivité thérapeutique, comme le montre l'exemple caricatural de l'hôpital Georges Pompidou, où les cas massifs d'infections nosocomiales du côté des patients s'ajoutent aux cas de suicide des personnels médicaux ou paramédicaux. 


\section{Affronter l'omerta sur la violence hospitalière}

Enfin, très clairement, ce qu'une volonté de "prendre soin » doit affronter, c'est la problématique structurelle d'une omerta hospitalière dont les services psychiatriques en particulier peuvent être le lieu.

En février 2011, je menais un entretien avec cinq personnes qui s'occupaient d'un ami atteint d'un déficit neurologique partiel, consécutif à une encéphalopathie. À la suite d'une tentative de suicide liée à la conscience qu'il avait de son état, ce dernier, parfaitement conscient, physiquement valide et totalement apte à la relation et à l'échange, avait été enfermé, mis à l'isolement et attaché pour des raisons qu'on peut sans peine qualifier de simplement punitives puisqu'elles n'avaient strictement aucune vocation thérapeutique, nul entretien n'étant mené avec le patient. Ses amis, qui constituaient sa seule famille, avaient alors été systématiquement mis à l'écart et privés d'information. C'est ce groupe d'amis solidaires et désemparés que j'ai rencontrés :

$\mathrm{CV}$ : Qu'est-ce qui vous a le plus marqués dans votre relation au monde hospitalier? $\mathrm{Z}$ : Le mépris à l'adresse du patient, parce qu'on le méprise, mais aussi à l'adresse des proches.

I : La souffrance de Philippe, c'est dur à vivre. La dignité de Philippe qui ne se plaint jamais. Comment il arrive à tenir là-dedans? C'est impressionnant.

M : Il n'est pas soigné. On le bourre de médocs, c'est tout. On n'est jamais ni dans la discussion, ni dans l'échange. Les soignants ne se justifient pas : ils pensent qu'ils n'ont pas à se justifier. Mais en même temps, on ne connaît pas cette institution, on la découvre.

Mo: Quand Philippe était encore dans sa chambre, il avait cette attitude suicidaire. L'infirmière me raccompagne à la porte. Je lui dis « On voudrait faire quelque chose pour lui ». Réponse: "On n'a pas besoin de vous ». Si on n'était pas là, je me demande ce qui se passerait pour Philippe. Quand je vois son voisin de chambre... On veut lui apporter quelque chose pour qu'il reste propre et conserve le minimum. En fait, on fait en partie le boulot des infirmiers et des aides-soignants. Si on n'était pas là, il y aurait une dégradation beaucoup plus rapide. Si on met les deux types d'intervention côte à côte, on sait que Philippe a besoin de nous.

F : Mépris, violence, déshumanisation, hostilité, toute-puissance. À l'égard de Philippe et de nous. Il y a eu tellement d' "échanges" qui ont été pour moi vraiment violents. Ce sont des choses qui t'atteignent.

J'essaie de ne jamais montrer de faiblesse, de ne pas craquer. Mais il y a des comportements hospitaliers qui sont pour moi violents. Tu rentres chez toi après, et tu t'effondres, parce que ce sont des choses très difficiles à mettre à distance.

Un sentiment d'incompréhension totale, des médicaux comme des paramédicaux. Je ne comprends pas ce manque total d'empathie, de prise en compte de la souffrance de l'entourage. Alors qu'on aurait tout intérêt à collaborer ensemble pour le bien-être de Philippe. C'est un parcours du combattant.

Le pire a été l'affaire des quatre points de suture. Je le retrouve suturé, et on me dit : «C'est une blessure banale au cuir chevelu ». Je dis : « Toutes les blessures au cuir chevelu ne saignent pas ». On me répond: «On ne va quand même pas faire déplacer la police scientifique pour Monsieur B. »

$\mathrm{Tu}$ demandes des informations, et on te raconte toute une série de conneries. Tu insistes pour voir des médecins, et il y a une psychiatre qui te dit "S'il fallait prévenir les familles dès qu'il y a le moindre souci ... On a d'autres patients à s'occuper".

C'est une illustration de la façon de traiter l'entourage : il n'y a jamais eu un mot d'excuse de la part des soignants. Quand on ose te dire qu'on l'a retrouvé comme ça au petit matin, on essaie de te faire avaler une couleuvre. Je pense qu'il s'est fait 
battre, peut-être par un patient. Au même moment, il y avait eu un autre problème plus grave à Tourcoing.

Et puis, on lui achète des pantalons, et ils disparaissent : "C'est parti à Bailleul ou avec les draps, vous savez comment est Monsieur B ». "On va faire un inventaire". «Vous avez appelé la lingerie à Bailleul ? ». « Ah ben ça, c'est Monsieur B ».

$\mathrm{I}:$ Il y a une dimension punitive. On punit Philippe, et on punit $\mathrm{F}$ qui s'occupe de lui. On enferme les vêtements sales ou trempés dans le sac, et c'est à $\mathrm{F}$ d'y aller pour les chercher et les laver. Et on lui dit : " Il est dans la provoc, il fait ça exprès ».

$\mathrm{Z}$ : Il est aussi puni de sortie. Il ne faut pas lui donner la possibilité de la lutte.

Mo : Ils lui mettent sans cesse des affaires qui ne lui appartiennent pas. Et la perm supprimée, c'est une punition: Philippe avait essayé de démonter une fenêtre pour s'échapper.

F : Une autre fois, la visite a été supprimée le 31 décembre: Philippe était à l'isolement pour s'être déshabillé dans le réfectoire.

F : Il y a une frayeur de la douche chez Philippe : les jets de douche provoquent des cris, au point de le trouver tout habillé sous la douche, ce sont des comportements liés à l'hygiène. Et je retrouve les fringues trempées dans le sac de linge sale.

$\mathrm{Z}$ : Parfois aussi, ils l'ont foutu habillé sous la douche.

Mo: Dans l'autre institution qui doit le prendre en charge, se produit un glissement progressif : il y a des analogies entre les institutions parce que c'est ce qu'il y a de plus simple pour eux. C'est pourquoi il y a une vraie hostilité contre nous et contre Philippe dans cette autre institution. Il y est rentré pour une période d'essai de six semaines. Et du coup, $\mathrm{F}$ a été très prise et a eu beaucoup moins de disponibilité. Il y a eu un coup de fil de la psychiatre : «Mme $C$ vous convoque pour faire le point ».

$\mathrm{Z}$ : Avec un comportement infantilisant vis-à-vis de l'entourage.

F : La lettre disait: "Mme C voudrait vous voir. On a constaté que vous étiez toujours angoissée, on veut en parler avec vous. On voudrait vous parler des difficultés de Monsieur B. Vous êtes convoquée tel jour à telle heure. " C'est un courrier d'assignation, pas d'ouverture à la discussion.

Si j'étais toute seule, j'aurais jeté l'éponge : on en prend plein la gueule pour pas un rond. Ma présence déclenche leur hostilité : je suis soupçonnée, j'aurai toujours à me justifier de ma relation avec Philippe. Je ne suis ni sa mère, ni sa compagne, ni sa sœur. C'est une relation de vingt ans, pour qui j'ai une affection immense, et il faut toujours que je me justifie.

Mo: La psychiatre veut considérer ton comportement comme pathologique, en jouant sur ce qu'elle sent être tes faiblesses.

$\mathrm{F}:$ Je ne peux tenir que parce que je ne suis pas seule.

$\mathrm{Z}$ : On a toujours l'impression de les déranger.

En mars 2016, la Contrôleure générale des lieux de privation de liberté, Adeline Hazan, dérogeant aux règles de préséance ordinaires, rend publiques les recommandations d'un rapport qu'elle n'a pas encore adressé au gouvernement. Il concerne le Centre psychothérapique de l'Ain à Bourg-en-Bresse, où la constance de pratiques indignes à l'égard des patients est pour la première fois dénoncée.

Mais, quelles que soient les horreurs qu'elle signale, elle est moins choquée par ce qu'elle a vu que par le total silence des institutions de contrôle qui l'ont précédée :

Je suis sidérée que l'Agence régionale de santé, que la Haute Autorité de santé, que les différentes commissions départementales, toutes ces structures qui sont venues ces dernières années, voire pour certaines ces dernières semaines, n'aient pas observé ce que notre mission a vu. Et qu'elles n'aient en tout cas pas réagi. Cela me laisse sans voix. ${ }^{11}$

Ce dispositif de black-out recouvre bel et bien ce que le philosophe René Girard, mort en novembre 2015, qualifiait de "violence mimétique ». La relégation ne constitue jamais seulement un isolement. Elle produit au contraire du commun: celui qui place le 
relégué à la merci des membres d'une institution, et l'inscrit donc dans une nouvelle communauté où son statut l'expose à la soumission. Et, à l'omerta qui soude de façon circulaire les pratiques de cette communauté, vient se surajouter un cercle concentrique plus large: celui des responsables administratifs et politiques qui l'autorisent. La double épaisseur de ce silence est comme une double muraille, rendue quasi infranchissable pour celui qui se trouve plongé au centre de cette concentricité.

L'éthique du care pose une affirmation de la vulnérabilité et de la dépendance comme principes fondateurs de l'humanité. Une telle affirmation recouvre bien une réalité anthropologique : celle que Hume mettait en évidence au XvIII ${ }^{e}$ siècle dans le Traité de la nature humaine, en affirmant l'interdépendance entre les hommes comme spécificité au sein de la nature. Et celle qu'affirmait Rousseau dans une morale de la "pitié ", considérant celle-ci comme ce qui unit les hommes au travers de la souffrance.

41 Mais la violence est tout aussi caractéristique, et tout aussi spécifique de l'humanité. Et, comme l'exposait déjà la pensée kantienne de l'histoire, les hommes ne peuvent progresser qu'à partir du conflit et de la rivalité : c'est ce qu'il mettait en œuvre à travers le concept d' «insociable sociabilité » : c'est la tension liée à l'insociabilité qui pousse les hommes à se surpasser, à inventer, à créer.

Ce que semble méconnaître l'idéologie du care, c'est précisément ce paradoxe anthropologique d'une tension constitutive. Et ce faisant, elle reproduit pour une large part un discours religieux de l'universel et de la compassion qui pose un déni non pas seulement sur la réalité de la violence, mais sur l'effectivité du désir de violence comme dynamique vitale. Or ce déni, dans l'histoire même des systèmes religieux, a été le vecteur des plus grandes violences. Les théoriciens de l'Inquisition, comme ses acteurs, portaient une parole de l'amour et de la charité et n'ont cessé de pleurer devant le martyre du Christ et de célébrer les souffrances de tous les saints. De même, les idéologies victimaires contemporaines produisent les plus redoutables arguments sécuritaires justifiant les violences policières.

Plutôt que la sollicitude, on pourra alors choisir la sollicitation : celle qui pousse à susciter en l'autre sa force plutôt que sa faiblesse. Pour notre part, c'est le sens que nous donnons à l'attention portée à l'autre dans le travail d'une philosophie de terrain.

\section{NOTES}

1. Layla Raïd, «Care et politique chez Joan Tronto » in P. Molinier, S. Laugier, P. Paperman (dir.), Qu'est-ce que le care?, Paris, Payot, 2009, p. 68.

2. Joan Tronto, "Aristote, care et politique " in .C. Lefève, L. Benaroyo, F. Worms (dir.), Les Classiques du soin, PUF, 2015, p. 37.

3. Ibid., p. 36.

4. Joan Tronto, "Care démocratique et démocratie du care " in Dir. P. Molinier, S. Laugier, P. Paperman, Qu'est-ce que le care ?, Payot, 2009, pp. 37, 38, 42.

5. Pascale Molinier, Introduction, in P. Molinier, S. Laugier, P. Paperman (dir.), Qu'est-ce que le care?, Payot, 2009, p. 12. 
6. Joan Tronto, «Care démocratique et démocratie du care» in P. Molinier, S. Laugier, P. Paperman (dir.), Qu'est-ce que le care ?, Payot, 2009, p. 45.

7. Evelyn Nakano-Glenn, «Le travail forcé: citoyenneté, obligation statutaire et assignation des femmes ", in P. Molinier, S. Laugier, P. Paperman (dir.), Qu'est-ce que le care?, Payot, 2009, p. 127-128.

8. Bridget Anderson, Doing the Dirty work? The Global Politics of Domestic Labour, Zed Books, 2000, p. 41.

9. Ibid., p. 50 .

10. Ibid., p. 49.

11. Eric Favereau, «L'enfer derrière les portes », cité par le numéro du 25 mars 2016 du journal.

\section{AUTHOR}

\section{CHRISTIANE VOLLAIRE}

Christiane Vollaire est philosophe, membre du Comité de rédaction des revues Pratiques et Chimère, et membre du Programme Non-lieux de l'exil (EHESS-Inalco) 